NoTe

\title{
Prediction of late (24-hour) radioactive iodine uptake using early (3-hour) uptake values in Japanese patients with Graves' disease
}

\author{
Yoshinori Osaki, Kanako Sakurai, Zenei Arihara, Masahiro Hata and Hiroshi Fukazawa \\ Department of Endocrinology and Metabolism, Federation of National Public Service and Affiliated Personnel Mutual Aid \\ Associations, Suifu Hospital, Mito 311-4141, Japan
}

\begin{abstract}
Measurement of 24-hour radioactive iodine uptake (RAIU), which is commonly used to calculate the dose of radioiodine (RI) therapy, cannot be accomplished in a single day. The purpose of this study was to predict 24-hour RAIU from 3-hour RAIU in Japanese patients with Graves' disease, and to investigate other factors that could be used to predict 24-hour RAIU. A total of 66 Japanese patients (14 men and 52 women; age, 17-83 years) with Graves' disease who had undergone both 3-hour and 24-hour ${ }^{123}$ I RAIU measurements between January 2006 and September 2011 were included in this study. Stepwise multiple regression analyses were performed in order to identify factors that could be used to predict 24-hour RAIU. The investigated factors were gender, age, thyroid volume, TSH, free thyroxine (FT4), free triiodothyronine (FT3), serum creatinine, second generation assay TSH receptor antibody (TRAb2), antithyroid drugs discontinuation period (ADP), iodine restriction period and 3-hour RAIU. The ADP was converted to an ordinal scale ADP score (ADPS) for multiple regression analyses. Multiple regression analyses showed that 3-hour RAIU $(P<0.001)$, FT3 $(P<0.001)$ and ADPS $(P<0.001)$ were statistically significant predictive factors of 24-hour RAIU. The relationship between 24-hour RAIU (LU) and 3-hour RAIU (EU), FT3 and ADPS was: LU $=11.5+29.1 \times \log _{10}$ EU $+23.0 \times \log _{10}$ FT3 $-2.7 \times$ ADPS $(\mathrm{r}=0.82, P<0.001)$. The present results indicate that prediction of LU from EU, FT3 and ADPS is feasible in Japanese patients with Graves' disease.
\end{abstract}

Key words: Graves’ disease, Hyperthyroidism, ${ }^{123}$ I-Radioactive iodine uptake, Radioiodine therapy, Japanese

RADIOACTIVE IODINE UPTAKE (RAIU) is widely used in clinical practice for diagnosis of thyroid disease. Iodine-123 $\left({ }^{123} \mathrm{I}\right)$ is used for differential diagnosis of hyperthyroidism and calculation of the therapeutic dose for iodine-131 $\left({ }^{131} \mathrm{I}\right)$ therapy in Graves' disease. Twenty-four-hour RAIU is commonly used to calculate the dose for radioiodine (RI) therapy; however, it is inconvenient because 2 days are needed for the 24-hour RAIU measurement. To this end, predictions of late (24-hour) RAIU using early (3- to 6-hour) RAIU with either ${ }^{131}$ I or ${ }^{123}$ I in patients with Graves' disease who live in areas where people take less iodine

Submitted Oct. 5, 2011; Accepted Nov. 4, 2011 as EJ11-0279

Released online in J-STAGE as advance publication Nov.18, 2011

Correspondence to: Yoshinori Osaki, M.D., Department of Endocrinology and Metabolism, Federation of National Public Service and Affiliated Personnel Mutual Aid Associations, Suifu Hospital, 1-1 Akatsuka, Mito 311-4141, Japan

E-mail: oosaki-tuk@umin.ac.jp than Japanese have been reported [1-4]. Three-hour RAIU is commonly used as early RAIU in Japan, but to our knowledge prediction of 24-hour RAIU using 3-hour RAIU values in Japanese patients with Graves' disease has not been reported. The purpose of the present study was to predict 24-hour RAIU from 3-hour RAIU values in Japanese patients with Graves' disease, and to investigate other factors that could be used to predict 24-hour RAIU.

\section{Materials and Methods}

\section{Subjects}

The present study enrolled 75 Japanese patients (17 men and 58 women; age, 17-83 years) with Graves' disease who had undergone both 3-hour and 24-hour ${ }^{123}$ I RAIU measurements in our hospital between January 2006 and September 2011. The diagnostic criteria for Graves' disease were based on the Japan Thyroid 
Association guideline for diagnosis of Graves' disease [5]. Treatment with antithyroid drugs (ATD) such as methimazole (MMI) and propylthiouracil (PTU) was discontinued for at least 3 days before RAIU measurement [6]. Dietary iodine restriction was initiated at least 3 days before RAIU measurement [7]. Dietary iodine restriction was performed through avoidance of the following foods: seaweed, seaweed soup and iodinated eggs. Patients who had received RI therapy or thyroidectomy were included in the present study. Of the 75 patients with Graves' disease who had undergone both 3-hour and 24-hour ${ }^{123}$ I RAIU measurements, 9 patients were excluded for the following two reasons: either dietary iodine restriction was continued for only 1 day $(n=3)$ or ATD was administered between diagnostic 3-hour RAIU measurement and RI therapeutic 24-hour RAIU measurement because adverse effects from ATD treatment required a change in treatment from ATD to RI therapy $(n=6)$. A total of 66 patients (14 men and 52 women; age, 17-83 years) were included in the present analyses. These 66 patients included 4 patients who had received RI therapy and 2 patients who had received thyroidectomy.

\section{Measurements}

Thyroid function was assessed by measuring serum free triiodothyronine (FT3) ("Vitros" FT3II kit, OrthoClinical Diagnostics K.K., Tokyo, Japan; reference range, 2.35-4.48pg/mL), free thyroxine (FT4) ("Vitros" FT4 kit, Ortho-Clinical Diagnostics; reference range, 0.81-1.58ng/dL) and TSH ("Vitros" TSH kit, OrthoClinical Diagnostics; reference range, 0.46-4.68 $\mu \mathrm{IU} /$ $\mathrm{mL}$ ). TSH binding inhibitory immunoglobulin (TBII) was measured by either a TSH receptor antibody (TRAb) first generation assay kit (TRAb "Cosmic" III, Cosmic Corp., Tokyo, Japan) or a TRAb second generation assay kit (DYNO test TRAb Human kit "YAMASA", Yamasa Corp., Tokyo, Japan). For statistical analyses, the first generation TRAb (TRAb1) value was converted to the second generation TRAb (TRAb2) value using the following equation: TRAb2 $=$ TRAb1 $\times 0.30+0.16$ [8]. Thyroid volume was measured as reported previously [9]. Maximum width (W), maximum thickness (T) and maximum length (L) were measured in both right (r) and left (l) lobes of the thyroid gland. Thyroid volume was calculated by the following equation: thyroid volume $=(\mathrm{rW} \times \mathrm{rT} \times \mathrm{rL}+\mathrm{lW} \times \mathrm{lT} \times \mathrm{lL}) \times 0.70$. The factor 0.7 was obtained by the comparison of thyroid volume measured by this method and actual thy- roid volume after thyroidectomy [9]. For measurement of RAIU, all patients ingested 2 capsules that contained 7.4 MBq of ${ }^{123}$ I. RAIU was measured with a scintillation probe with a NaI crystal (E.CAM, Toshiba Medical Systems Corp., Tochigi, Japan) at a distance of $15 \mathrm{~cm}$ from the thyroid. Radiation from a ${ }^{123}$ I standard that was placed in a thyroid uptake phantom was counted for 20 seconds. The ${ }^{123}$ I standard was the same volume and activity as the dose administered to the patients. Patients were counted with the scintillation probe for 20 seconds at both 3 and 24 hours after ${ }^{123}$ I ingestion. The thyroid ${ }^{123}$ I uptake value was calculated using the following equation: RAIU $(\%)=$ (neck count - background) / (standard count - background) $\times 100$.

\section{Statistical Analysis}

Forward-backward stepwise multiple regression analyses were performed to investigate factors that could be used to predict 24-hour RAIU. Factors investigated were gender, age, thyroid volume, TSH, FT4, FT3, serum creatinine (Cre), TRAb2, ATD discontinuation period (ADP), iodine restriction period and 3-hour RAIU. For multiple regression analyses, ADP was assigned as follows: 3 days, 4 days, 5 days, 6 days, and either 7 or more days or no ATD administration were assigned to scores of $0,1,2,3$ and 4, respectively. The ADP score (ADPS) was included in the model. Before multiple regression analyses, a logarithmic transformation was performed for variables with nonnormal distributions in order to fit the data into a normal distribution. Normal distribution was tested with the Shapiro-Wilk test. A $P$-value $<0.05$ was considered statistically significant. All data with normal distributions were expressed as mean \pm standard deviation (SD) and all data with non-normal distributions were expressed as median \pm interquartile range (IQR). All statistical analyses were performed using SPSS version 16.0 software (SPSS Japan, Tokyo, Japan).

\section{Results}

Table 1 shows clinical characteristics of the 66 patients with Graves' disease. Twenty-four-hour RAIU values were greater than 3-hour RAIU values in all patients. TRAb1 values of 9 patients were converted to TRAb2 values by the aforementioned equation [8]. Fig. 1 shows the relationship between 3-hour and 24-hour RAIU analyzed by simple regression analyses ( $\mathrm{r}=0.70, P<0.001$, Fig. 1 ). As shown in Table 2, mul- 
Table 1 Characteristics of patients with Graves' disease

\begin{tabular}{lc}
\hline & Total (n = 66) \\
\hline Gender (Male / Female) & $14 / 52$ \\
Age (years) & $49.2 \pm 15.8^{*}$ \\
Thyroid volume (g) & $48.8 \pm 38.7^{* *}$ \\
TSH ( $\mu \mathrm{IU} / \mathrm{mL})$ & $0.00 \pm 0.01^{* *}$ \\
FT4 (ng/dL) & $2.34 \pm 2.32^{* *}$ \\
FT3 (pg/mL) & $8.23 \pm 9.73^{* *}$ \\
Cre (mg/dL) & $0.48 \pm 0.13^{*}$ \\
TRAb2 (IU/L) & $8.5 \pm 17.9^{* *}$ \\
ATD discontinuation period (days) ${ }^{\ddagger}$ & $5.0 \pm 5.0^{* *}$ \\
Iodine restriction period (days) & $6.0 \pm 4.0^{* *}$ \\
3-hour RAIU (\%) & $40.0 \pm 27.4^{* *}$ \\
24-hour RAIU (\%) & $(9.3-87.3)^{\dagger}$ \\
\hline
\end{tabular}

${ }^{*}$ Data indicate mean \pm standard deviation. ${ }^{* *}$ Data indicate median \pm inter quartile range. ${ }^{\ddagger} 31$ patients (5 men and 26 women) did not receive antithyroid drugs. ${ }^{\dagger}$ Range. FT4, free thyroxine; FT3, free triiodothyronine; Cre, serum creatinine; TRAb2, second generation TSH receptor antibody; ATD, antithyroid drugs; RAIU, radioactive iodine uptake.

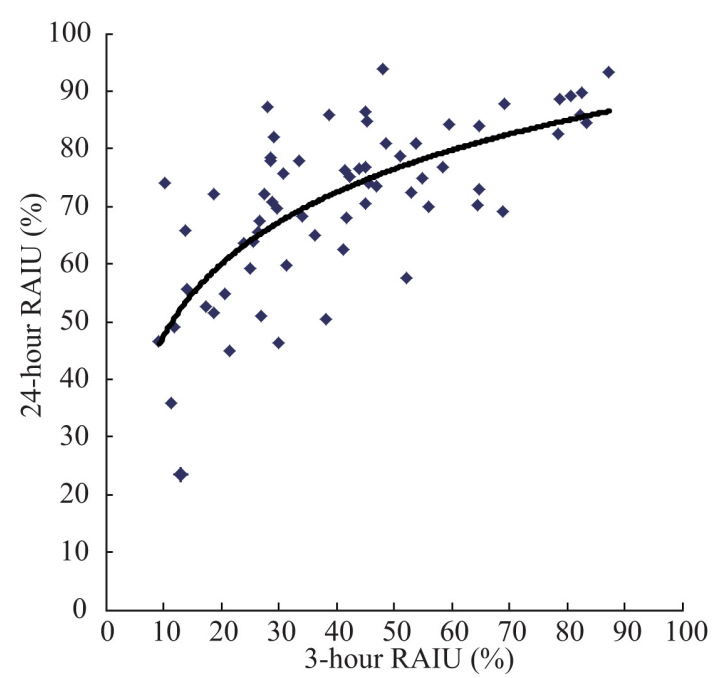

Fig.1 Relationship between 3-hour and 24-hour RAIU analyzed by simple regression analyses. Equation for the curve: $\mathrm{LU}=5.9+$ $41.5 \times \log _{10} \mathrm{EU}(\mathrm{r}=0.70, P<0.001)$. RAIU, radioactive iodine uptake; EU, 3-hour RAIU; LU, 24-hour RAIU.
Table 2 Multiple regression analyses concerning prediction of 24-hour RAIU

\begin{tabular}{lcc}
\hline Dependent variable & $R^{2}$ & \multicolumn{2}{c}{ Overall $P$ value } \\
\hline 24-hour RAIU & 0.67 & $<0.001$ \\
\hline \hline Independent variables & B coefficient $(95 \%$ CI $)$ & $P$ value \\
\hline $\log _{10}$ (3-hour RAIU) $^{*}$ & $29.1(19.5$ to 38.6) & $<0.001$ \\
$\log _{10}$ (FT3) ${ }^{*}$ & $23.0(14.7$ to 31.3) & $<0.001$ \\
ADPS & -2.7 (-4.1 to -1.3$)$ & $<0.001$ \\
Gender & NA & 0.266 \\
Age & NA & 0.452 \\
$\log _{10}$ (Thyroid volume) & NA & 0.291 \\
$\log _{10}$ (TSH) & NA & 0.497 \\
$\log _{10}$ (FT4) & NA & 0.096 \\
Cre & NA & 0.282 \\
$\log _{10}$ (TRAb2) & NA & 0.156 \\
$\log _{10}$ (Iodine restriction period) & NA & 0.733 \\
\hline
\end{tabular}

${ }^{*}$ Statistically significant predictive factors. CI, confidence interval; NA, not available; RAIU, radioactive iodine uptake; FT3, free triiodothyronine; ADPS, antithyroid drugs discontinuation period score; FT4, free thyroxine; Cre, serum creatinine; TRAb2, second generation TSH receptor antibody.

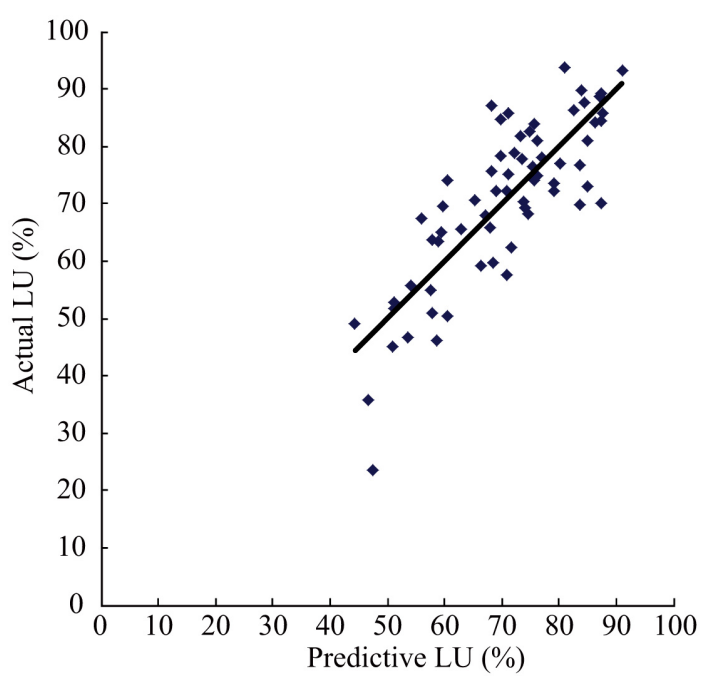

Fig.2 Relationship between predictive and actual 24-hour RAIU analyzed by multiple regression analyses. Predictive equation of $\mathrm{LU}: \mathrm{LU}=11.5+29.1 \times \log _{10} \mathrm{EU}+23.0 \times \log _{10}$ FT3 $2.7 \times$ ADPS $(\mathrm{r}=0.82, P<0.001)$. RAIU, radioactive iodine uptake; LU, 24-hour RAIU; EU, 3-hour RAIU; FT3, free triiodothyronine; ADPS, antithyroid drugs discontinuation period score.

$=0.156)$ and iodine restriction period $(P=0.733)$, were not statistically significant predictive factors of 24 -hour RAIU. The relationship between 24-hour RAIU (LU), 3-hour RAIU (EU), FT3 and ADPS was as follows: LU $=11.5+29.1 \times \log _{10} \mathrm{EU}+23.0 \times \log _{10} \mathrm{FT} 3-2.7$

$\times \operatorname{ADPS}(r=0.82, P<0.001$, Fig. 2). tiple regression analyses revealed that 3-hour RAIU ( $P$ $<0.001)$, FT3 $(P<0.001)$ and ADPS $(P<0.001)$ were statistically significant predictive factors of 24-hour RAIU. All other factors, including gender $(P=0.266)$, age $(P=0.452)$, thyroid volume $(P=0.291)$, TSH $(P$ $=0.497)$, FT4 $(P=0.096)$, Cre $(P=0.282)$, TRAb2 $(P$ 
Table 3 Comparison of regression equations for predicting late (24-hour) RAIU from early (3- to 6-hour) RAIU values in patients with Graves’ disease.

\begin{tabular}{|c|c|c|c|c|}
\hline Source & $\mathrm{N}$ & Method & Equation & Correlation \\
\hline Authors' equation & 66 & 3-hour EU with ${ }^{123}$ I, FT3 and ADPS & $\mathrm{LU}=11.5+29.1 \log _{10} \mathrm{EU}+23.0 \log _{10} \mathrm{FT} 3-2.7 \mathrm{ADPS}$ & $r=0.82$ \\
\hline Authors' equation & 66 & 3-hour EU with ${ }^{123} \mathrm{I}$ & $\mathrm{LU}=5.9+41.5 \log _{10} \mathrm{EU}$ & $r=0.70$ \\
\hline Hayes et al. [1] & 27 & 3- to 6-hour EU with ${ }^{131} \mathrm{I}$ & $\mathrm{LU}=-55.7+73.2 \log _{10} \mathrm{EU}$ & $r=0.94$ \\
\hline Vemulakonda et al. [3] & 35 & 4-hour EU with ${ }^{123} \mathrm{I}$ & $\mathrm{LU}=-38.62+65.22 \log _{10} \mathrm{EU}$ & $r=0.85$ \\
\hline Morris et al. [4] & 61 & 4-hour EU with ${ }^{123} \mathrm{I}$ & $\mathrm{LU}=23.63+97.25(\% \mathrm{EU})$ & $r=0.76$ \\
\hline Morris et al. [4] & 21 & 6-hour EU with ${ }^{123} \mathrm{I}$ & $\mathrm{LU}=19.09+94.38(\% \mathrm{EU})$ & $r=0.94$ \\
\hline
\end{tabular}

$\mathrm{N}$, number of patients; RAIU, radioactive iodine uptake; EU, early RAIU; LU, 24-hour RAIU; FT3, free triiodothyronine; ADPS, antithyroid drugs discontinuation period score.

\section{Discussion}

Table 3 compares the present early-to-late RAIU conversion equation and several other early-to-late RAIU conversion equations that have been previously reported by other investigators $[1,3,4]$. These previously reported equations suggest a stronger correlation between early RAIU and late RAIU than the present equation, which employs only 3-hour RAIU. However, the present equation, which employs 3-hour RAIU, FT3 and ADPS, suggests a correlation between early RAIU and late RAIU that is as strong as the other authors' equations. Reasons for the differences in strength of correlation between early and late RAIU are uncertain, but could be attributable to the time of the early RAIU measurement, to iodine intake before RAIU measurement, or to ethnicity.

Several other investigators have reported that patients with hyperthyroidism or Graves' disease have "rapid turnover" (24-hour RAIU value is less than the 3- to 4-hour RAIU value; typically characterized by a significantly increased early RAIU value) $[3,4,10$, 11]. Furthermore, Marcocci et al. [10] and Atkay et al. [11] reported that rapid turnover is a statistically significant predictor of RI therapy failure because of rapid clearance or turnover of RI from the thyroid gland. RI therapy failure rates were reported to be $55 \%$ in 87 Graves' disease patients and 48\% in 67 hyperthyroidism patients with rapid turnover, compared to $21 \%$ in 187 Graves' disease patients and 11\% in 366 hyperthyroidism patients without rapid turnover [10, 11]. The prevalence of rapid turnover was reported to be $32 \%$ in 274 patients with Graves' disease by Marcocci et al. [10], 15\% in 433 patients with hyperthyroidism (including 379 patients with Graves' disease) by Atkay et al. [11], 17\% in 69 patients with Graves' disease by Vemulakonda et al. [3], and 12\% in 148 patients with Graves' disease by Morris et al. [4]. In the present study, none of the patients with Graves' disease had rapid turnover. Morris et al. [4] also reported that Graves' disease patients with 4-hour RAIU values of $77 \%$ or greater also should obtain 24-hour RAIU values, probably due to rapid turnover. However, only 7 of 66 patients had 3-hour RAIU values greater than $77 \%$ in the present study, and none of those 7 patients had rapid turnover. The reasons for these differences in rates of rapid turnover are uncertain, but they could be attributable to iodine intake before RAIU measurement or to ethnicity.

In the present multiple regression equation, an increase in the ADP resulted in a decrease of the predictive 24-hour RAIU value. Kubota et al. [6] reported that 2-day ATD discontinuation sufficiently increased the 24-hour RAIU. Therefore, an increase in the ADP may not decrease the 24-hour RAIU, but may increase the 3-hour RAIU compared to the 24-hour RAIU. Atkay et al. [11] also estimated that administration of ATD may alter RI thyroidal kinetics and increase RI turnover. In the present study, 35 of the 66 patients with Graves' disease had received ATD, but none of the patients exhibited rapid turnover. The relationship between ATD administration and rapid turnover remains controversial.

In the present study, multiple regression analyses showed that not only 3-hour RAIU but also FT3 and ADPS were statistically significant predictive factors of 24-hour RAIU. The present study included only 14 men with Graves' disease, and no patients with rapid turnover were observed. Therefore, further studies may be required to determine a more accurate equation for prediction of 24-hour RAIU.

In conclusion, the present results in Japanese patients 
with Graves' disease indicate that unless patients have rapid turnover, prediction of 24-hour RAIU using 3-hour RAIU, FT3 and ADPS is feasible. With this prediction equation both measurement of RAIU and calculation of the RI therapy dose can be achieved in a single day.

\section{References}

1. Hayes AA, Akre CM, Gorman CA (1990) Iodine131 treatment of Graves' disease using modified early iodine-131 uptake measurements in therapy dose calculations. J Nucl Med 31: 519-522.

2. Hennessey JV, Berg LA, Ibrahim MA, Markert RJ (1995) Evaluation of early (5 to 6 hours) iodine 123 uptake for diagnosis and treatment planning in Graves' disease. Arch Intern Med 155: 621-624.

3. Vemulakonda US, Atkins FB, Ziessman HA (1996) Therapy dose calculation in Graves' disease using early I-123 uptake measurements. Clin Nucl Med 21: 102105.

4. Morris LF, Waxman AD, Braunstein GD (2000) Accuracy considerations when using early (four- or sixhour) radioactive iodine uptake to predict twenty-fourhour values for radioactive iodine dosage in the treatment of Graves’ disease. Thyroid 10: 779-787.

5. Japan Thyroid Association (2010) Guidelines for the diagnosis of thyroid disease 2010. http://www.japanthyroid.jp/doctor/guideline/english.html.

6. Kubota S, Ohye H, Yano G, Nishihara E, Kudo T, Ito M, Fukata S, Amino N, Kuma K, Miyauchi A (2006) Two-day thionamide withdrawal prior to radioiodine uptake sufficiently increases uptake and does not exacerbate hyperthyroidism compared to 7-day withdrawal in Graves’ disease. Endocr J 53: 603-607.

7. Kato Y, Kimura H, Sato K, Kusakabe K, Takano K (2008) Optimal Restriction Period of Dietary Iodine Intake before Radioiodine Therapy for Patients with Graves' Disease. Journal of Tokyo Women's Medical University 78: 468-473 (In Japanese).

8. Takeoka K, Hidaka Y, Hanada H, Nomura T, Izumi Y, Takano T, Amino N (2003) Evaluation of second generation kit for a TSH receptor antibodies (TRAb) detection. Igaku to Yakugaku 49: 283-291 (In Japanese).

9. Murakami Y, Takamatsu J, Sakane S, Kuma K, Ohsawa $\mathrm{N}$ (1996) Changes in thyroid volume in response to radioactive iodine for Graves’ hyperthyroidism correlated with activity of thyroid-stimulating antibody and treatment outcome. J Clin Endocrinol Metab 81: 32573260.

10. Marcocci C, Gianchecchi D, Masini I, Golia F, Ceccarelli C, Bracci E, Fenzi GF, Pinchera A (1990) A reappraisal of the role of methimazole and other factors on the efficacy and outcome of radioiodine therapy of Graves' hyperthyroidism. J Endocrinol Invest 13: 513-520.

11. Aktay R, Rezai K, Seabold JE, Bar RS, Kirchner PT (1996) Four- to twenty-four-hour uptake ratio: an index of rapid iodine-131 turnover in hyperthyroidism. $\mathrm{J} \mathrm{Nucl}$ Med 37: 1815-1819. 